\title{
QUADRATIC CURVATURE ENERGIES IN THE 2-SPHERE
}

\author{
JOSU ARROYO, ÓSCAR J. GARAY ${ }^{\bowtie}$ and JOSE MENCÍA
}

(Received 9 September 2009)

\begin{abstract}
The classical variational analysis of curvature energy functionals, acting on spaces of curves of a Riemannian manifold, is extremely complicated, and the procedure usually can not be completely developed under such a degree of generality. Sometimes this difficulty may be overcome by focusing on specific actions in real space forms. In this note, we restrict ourselves to quadratic Lagrangian energies acting on the space of closed curves of the 2-sphere. We solve the Euler-Lagrange equation and show that there exists a two-parameter family of closed critical curves. We also discuss the stability of the circular critical points. Since, even for this class of energies, the complete variational analysis is quite involved, we use instead a numerical approach to provide a useful method of visualization of relevant aspects concerning uniqueness, stability and explicit representation of the closed critical curves.
\end{abstract}

2000 Mathematics subject classification: primary 53C20; secondary 58E30, 70H03, 74B05.

Keywords and phrases: elastic curves, Euler-Lagrange equations, curvature energy functionals.

\section{Introduction}

Assume that $\gamma:[0,1] \rightarrow \mathbf{M}$ is an immersed curve in a Riemannian manifold and denote its curvature by $\kappa$. Curves which are minimizers (under suitable boundary conditions) of energies depending quadratically on $\kappa$,

$$
\mathcal{F}(\gamma)=\int_{\gamma}\left(\varepsilon \kappa^{2}+\lambda \kappa+\mu\right) d s,
$$

with $\varepsilon, \lambda, \mu \in \mathbb{R}$, have often been employed to construct useful models in physics. Thus, if $\varepsilon=\lambda=0$, then $\mathcal{F}$ is basically the length of the curve, and unit speed critical points are nothing but geodesics, the trajectories of particles when no external forces act on them. If $\varepsilon=0$, then, according to a well-known model [18], critical curves of $\mathcal{F}$ represent relativistic particles (both massive and massless) in pseudo-Riemannian ambient spaces (see also [5] and the references therein). On the other hand, if $\lambda=0$ critical curves of (1.1) correspond to the Euler-Bernoulli classical model for the elastic

This research has been partially supported by grants GIC07/58-IT-256-07 of Gobierno Vasco and MTM2007-61990 of Ministerio de Ciencia e Innovación. Spain.

(c) 2010 Australian Mathematical Publishing Association Inc. 0004-9727/2010 \$16.00 
curves (or simply, elasticae) which has been widely studied in real space forms by means of various approaches $[6,8,10,11,13,14,16]$. In this setting, extremals of $\mathcal{F}$ can be interpreted in physics not only in terms of the elasticity theory [12], but also in connection with the effective action of the relativistic kink model around the solitonic solution [9]. Elasticae in Riemannian manifolds with nonconstant curvature are not so well understood. The analysis of the elasticae in Lie groups equipped with bi-invariant Riemannian metrics was recently initiated in [17], where the authors focused on the group of rotations of $\mathbb{R}^{3}$ with a view to applications in robotics, engineering and computer graphics.

Our purpose here is to focus the variational problem associated with the energy $\mathcal{F}$ given in (1.1) when $\varepsilon^{2}+\lambda^{2} \neq 0$. Of course, boundary conditions need to be established and we will consider the energy $\mathcal{F}$ acting on the space of closed curves. As a consequence of the rotation index theorem, closed critical points of (1.1) in $\mathbb{R}^{2}$ are nothing but the closed planar elasticae already known to Euler: circles and figureof-eight curves. In the 2 -sphere $\mathbb{S}^{2}(1)$ the situation is much more interesting. If $\varepsilon=0$, then it is easy to see that the only critical curves are circles [1]. Actually, taking in addition $\mu=1$ and considering $\mathcal{F}$ as acting on the space of closed embedded curves in $\mathbb{S}^{2}(1)$, one can use the Gauss-Bonnet formula to see the critical points of $\mathcal{F}$ as extremals of the length, for variations with fixed enclosed area (whose minimizers are circles as a consequence of the isoperimetric inequality). If $\lambda=0$, critical points of $\mathcal{F}$ are closed elasticae in $\mathbb{S}^{2}(1)$, which have been classified in [13] using classical techniques of the calculus of variations. When $\varepsilon=1, \mu=(\lambda / 2)^{2}$, then extremals of $\mathcal{F}$ correspond to elasticae which are circular at rest [4].

Arbitrary curvature energy actions on $\mathbb{S}^{2}(1)$ have been studied in [3], by extending the ideas contained in [13] (see also [2]). However, the program can not be fully developed for such a general choice of the Lagrangian. In this note we choose $\mu=0$ and $\varepsilon \lambda \neq 0$ in (1.1). Then, considering these parameters as Lagrange multipliers, extremals of $\mathcal{F}$ can be seen as critical points of the elastic energy for variations with fixed total curvature or, alternatively, as critical points of the total curvature for variations with constant elastic energy. Notice that, in the previous sentence, 'total curvature' can be replaced by 'enclosed area', for closed embedded curves. Observe also that there are no critical curves for the total curvature in $\mathbb{S}^{2}(1)$, since they must lie within the set of flat points of a surface [1]. The remaining choice $\mu \neq 0, \varepsilon \lambda \neq 0$ in (1.1) would impose an additional penalty on the length of the curve, and can be treated similarly.

The Euler-Lagrange equation associated with $\mathcal{F}$ can be expressed in terms of the curvature of the potential critical curves. In Section 2 we explicitly solve the EulerLagrange equation and find its periodic solutions. They give rise to corresponding curves which are the candidates to be closed extremals. In Section 3 we give a condition that these candidates must satisfy in order to close up, and show that there exists a two-parameter family of critical curves that fulfills it. The procedure described here allows us to obtain explicit parameterizations of the closed critical curves (at least by quadratures). The stability of the circular critical points for $\mathcal{F}$ is also analyzed in 
this section. Further formal investigation concerning uniqueness, classification and stability is extremely hard even for $\lambda=0$ [15]. In Section 4 we use a numerical approach to deal with these relevant aspects and gain some insight into the family of closed extremals for $\mathcal{F}$.

\section{Solving the Euler-Lagrange equation}

Let $\gamma(t)$ be regular closed curve in $\mathbb{S}^{2}(1)$, that is, $\gamma: \mathbb{S}^{1}(1) \rightarrow \mathbb{S}^{2}(1)$ is a $C^{\infty}$ immersion in the two-dimensional standard unit sphere. The arc-length parameter of $\gamma(t)$ is denoted by $s$. As usual, we denote by $\gamma(s)$ and $\kappa(s)$ the corresponding reparameterization and the curvature function of $\gamma(t)$, respectively. As already stated, we take $\mu=0$ and $\varepsilon \lambda \neq 0$ in (1.1), and, without loss of generality, we can choose $\varepsilon=1, \lambda>0$. We are concerned with the variational problem associated with the energy functional

$$
\mathcal{F}^{\lambda}(\gamma)=\int_{\gamma}\left(\kappa^{2}+\lambda \kappa\right) d s,
$$

acting on the space of closed immersed curves in $\mathbb{S}^{2}(1)$. The first variation formula of $\mathcal{F}^{\lambda}$ can be computed by using standard arguments, and the boundary operator vanishes identically for closed curves. Consequently, closed curves of $\mathbb{S}^{2}(1)$, which are critical for $\mathcal{F}^{\lambda}$, are characterized by the Euler-Lagrange equation (for details, see [3])

$$
2 \kappa_{s s}+\kappa^{3}+2 \kappa+\lambda=0
$$

where the subscript $s$ denotes differentiation with respect to the arc-length. Notice that geodesics are not critical points since $\lambda>0$. Circles of constant geodesic curvature

$$
\kappa_{o}=\varpi^{1 / 3}-\frac{2}{3} \varpi^{-1 / 3},
$$

where $\varpi=\left(\sqrt{\lambda^{2}+32 / 27}-\lambda\right) / 2$, are trivial closed solutions of (2.2). Assume, then, that $\kappa(s)$ is not constant. It is easy to see that a first integral of (2.2) is given by

$$
\kappa_{s}^{2}=\frac{1}{4} Q_{d}(\kappa)
$$

where $Q_{d}(\kappa):=d-\kappa^{4}-(\lambda+2 \kappa)^{2}$ and $d>0$ is a real constant. A phase portrait analysis would reveal that almost any solution of (2.4) is periodic. For our purposes, however, we will need to solve (2.4) explicitly. When solving $\int\left(d \kappa / Q_{d}(\kappa)^{1 / 4}\right)=$ $\pm 1 / 2 \int d s$, one can discuss the solutions of (2.4) in terms of the roots of the polynomial $Q_{d}(x)$, its multiple roots being those which affect the integrability. If $Q_{d}(x)$ has no multiple roots, then it admits two simple roots. Observe that $Q_{d}(x)$ takes its maximum at $\kappa_{o}$ (2.3). Thus, $Q_{d}(x)$ admits two simple roots $\alpha>\beta$ when $Q_{d}\left(\kappa_{o}\right)>0$. This happens precisely if

$$
d>d_{o}:=\left(\lambda+2 \kappa_{o}\right)\left(\lambda+\kappa_{o}\right)
$$


and in this case equation (2.4) reduces to

$$
\kappa_{s}^{2}=\frac{1}{4}(\alpha-\kappa)(\kappa-\beta)\left((\kappa-m)^{2}+n^{2}\right),
$$

where $m, n$ are determined by

$$
\begin{aligned}
& \alpha+\beta+2 m=0, \quad \alpha \beta+2 m(\alpha+\beta)+m^{2}+n^{2}=4, \\
& \alpha \beta\left(m^{2}+n^{2}\right)=\lambda^{2}-d, \quad 2 m \alpha \beta+(\alpha+\beta)\left(m^{2}+n^{2}\right)=-4 \lambda .
\end{aligned}
$$

Our first task will be to explore the set of solutions of (2.6). Remember that $\lambda>0$ and that $d$ satisfies (2.5). Then, by considering the Jacobi elliptic functions and invoking the formulas I.3.145 and I.3.147 of [7], one can see that the periodic solutions of (2.6) are given by

$$
\kappa(s)=\frac{(q \alpha+p \beta)-(q \alpha-p \beta) \operatorname{cn}(r s, M)}{(p+q)+(p-q) \operatorname{cn}(r s, M)},
$$

where $\mathrm{cn}(\cdot, \cdot)$ stands for the Jacobi elliptic cosine function and the parameters involved in (2.8) are determined by

$$
\begin{aligned}
& p^{2}=(m-\alpha)^{2}+n^{2}, \quad q^{2}=(m-\beta)^{2}+n^{2}, \\
& M=\frac{1}{2} \sqrt{\frac{(\alpha-\beta)^{2}-(p-q)^{2}}{p q}}, \quad r=\sqrt{p q} / 2 .
\end{aligned}
$$

Thus, the functions $\kappa(s)$ are periodic with period $\rho=4 K(M) / r$, where $K(M)$ denotes the complete elliptic integral of the first kind of modulus $M$.

\section{Closed critical points}

Next, we wish to determine under what conditions a curve of $\mathbb{S}^{2}(1)$, associated with a periodic solution to the Euler-Lagrange equation (2.2), is closed. For that purpose, we introduce a coordinate system adapted to the problem. Let $\kappa(s)$ be a periodic solution of (2.2) as given in (2.8) and denote its period by $\rho$. Take $\gamma(s)$ to be the curve in $\mathbb{S}^{2}(1)$ with curvature $\kappa(s)$ (which is unique up to isometries) and denote by $\{T(s), N(s)\}$ its Frenet frame. Then the vector field along $\gamma(s)$ defined by $\mathcal{J}=\kappa^{2} T+2 \kappa_{S} N$ can be extended to a Killing field on $\mathbb{S}^{2}(1)$, which we also denote by $\mathcal{J}(s)$. Choose geographic coordinates, $x(\theta, \phi)=(\cos \theta \sin \phi, \sin \theta \sin \phi, \cos \phi)$, such that the equator gives the only integral geodesic of $\mathcal{J}(s): x_{\theta}=b \mathcal{J}$. The advantages of choosing this coordinate system are various. For instance, not only will it be useful for numerical computations, but also it allows us to compute by quadratures the coordinates of a critical curve of $\mathcal{F}^{\lambda}, \gamma(s)=x(\theta(s), \phi(s))$, in terms of its curvature. To be more specific (for details, see $[2,13]$ ), we can write

$$
\begin{aligned}
& \theta_{s}(s)=\frac{\kappa^{2}}{b\left(d-(\lambda+2 \kappa)^{2}\right)}, \\
& b^{2}\left(d-(\lambda+2 \kappa)^{2}\right)=\sin ^{2} \phi .
\end{aligned}
$$


Hence, we can express the clousure condition for critical curves associated with periodic solutions of the Euler-Lagrange equation in a simple manner. In fact, defining the angular progression of $\gamma(s)$ by

$$
\Lambda^{\lambda}(d):=\sqrt{d} \int_{0}^{\rho} \frac{\kappa^{2}}{d-(\lambda+2 \kappa)^{2}} d s
$$

one can establish the following result.

PROPOSITION 3.1. Let $\gamma(s)$ be a curve in $\mathbb{S}^{2}(1)$ corresponding to a periodic solution of (2.2), $\kappa(s)$, with period $\rho$. Then $\gamma(s)$ is a closed critical curve for $\mathcal{F}^{\lambda}$ if and only if $\Lambda^{\lambda}(d) / 2 \pi \in \mathbb{Q}$.

Our next step will be to verify whether or not this condition is satisfied for any $\lambda>0$, and to examine the family of closed critical curves. We have the following proposition.

PROPOSITION 3.2. Let $\lambda$ be any positive real number. Then, for any pair of natural numbers $m, n \in \mathbb{N},(m, n)=1$, satisfying

$$
\frac{m}{n}<-\frac{2 \sqrt{d_{o}} \kappa_{o}}{\left(\lambda+2 \kappa_{o}\right) \sqrt{6 \kappa_{o}^{2}+4}},
$$

where $\kappa_{o}$ and $d_{o}$ are given by (2.3) and (2.5) respectively, there exists a closed critical curve of $\mathcal{F}^{\lambda}$ in $\mathbb{S}^{2}(1), \gamma_{m, n}(s)$, which closes up after traveling $n$ periods of its curvature and $m$ trips around the equator.

PROOF. We analyze the range of variation of the angular progression of $\gamma(s)$ when the curvature is given by (2.8). By using (2.4) and (3.2) we get

$$
\Lambda^{\lambda}(d)=4 \sqrt{d} \int_{\beta}^{\alpha} \frac{\kappa^{2} d \kappa}{\left(d-(\lambda+2 \kappa)^{2}\right) \sqrt{Q_{d}(\kappa)}},
$$

which is always positive since, from (2.4), $d \geq(\lambda+2 \kappa)^{2}$. Equality holds when the largest root of $Q_{d}$ vanishes and, therefore, $d=\lambda^{2}$. The behavior of $\Lambda^{\lambda}(d)$ as $d \rightarrow d_{o}$ can be studied by using the relations (2.7). One can check that

$$
\begin{aligned}
& \kappa_{o}+m=0, \quad \kappa_{o}^{2}+4 m \kappa_{o}+m^{2}+n^{2}=4, \\
& m \kappa_{o}^{2}+\kappa_{o}\left(m^{2}+n^{2}\right)=-2, \quad \kappa_{o}^{2}\left(m^{2}+n^{2}\right)=\lambda^{2}-d_{o} .
\end{aligned}
$$

Hence, as $d \rightarrow d_{o}$, we have $m=-\kappa_{o}, n^{2}=4+2 \kappa_{o}^{2}$ and $p^{2}=q^{2}=4 \kappa_{o}^{2}+n^{2}=$ $6 \kappa_{o}^{2}+4$. Also the period of the curvature, $\rho$, and the derivative of the longitude, $\theta_{s}(s)$, satisfy $\lim _{d \rightarrow d_{o}} \rho=\pi / r=4 \pi / p$ and $\lim _{d \rightarrow d_{o}} \theta_{s}(s)=-\left(\sqrt{d_{o}} \kappa_{o}\right) /\left(\lambda+2 \kappa_{o}\right)$, respectively. Therefore,

$$
\lim _{d \rightarrow d_{o}} \Lambda^{\lambda}(d)=-\left(\frac{4 \pi}{p}\right) \frac{\sqrt{d} \kappa_{o}}{\left(\lambda+2 \kappa_{o}\right)}=-\frac{4 \pi \sqrt{d_{o}} \kappa_{o}}{\left(\lambda+2 \kappa_{o}\right) \sqrt{6 \kappa_{o}^{2}+4}} .
$$


Analogously, after some lengthly computations,

$$
\begin{aligned}
& \lim _{\lambda \rightarrow+\infty} \kappa_{o}(\lambda)=-\infty, \quad \lim _{\lambda \rightarrow+\infty} \frac{\kappa_{o}^{3}(\lambda)}{\lambda}=-1, \quad \lim _{\lambda \rightarrow+\infty} \frac{d_{o}(\lambda)}{\lambda^{2}}=1, \\
& \lim _{\lambda \rightarrow 0} \kappa_{o}(\lambda)=0, \quad \lim _{\lambda \rightarrow 0} \frac{\kappa_{o}(\lambda)}{\lambda}=-\frac{1}{2}, \quad \lim _{\lambda \rightarrow 0} \frac{d_{o}(\lambda)}{\lambda^{2}}=0,
\end{aligned}
$$

and, consequently, we obtain

$$
\lim _{\lambda \longrightarrow 0} \Lambda^{\lambda}\left(d_{o}\right)=2 \pi, \quad \lim _{\lambda \longrightarrow+\infty} \Lambda^{\lambda}\left(d_{o}\right)=\sqrt{(2 / 3)} 2 \pi .
$$

On the other hand, notice that $\lim _{d \rightarrow+\infty}(-\beta / \alpha)=1$, and that $\alpha=O(\sqrt[4]{d}), n=$ $O(\sqrt[4]{d})$. Moreover, the function $f(\kappa)=\kappa^{2} /\left(d-(\lambda+2 \kappa)^{2}\right)$ takes its maximum value, $f(\alpha)=1 / \alpha^{2}$, at $\kappa=\alpha>0$. The previous considerations lead us to the following estimation: for $d$ large enough,

$$
\begin{aligned}
\Lambda^{\lambda}(d) & \leq \frac{4 \sqrt{d}}{n \alpha^{2}}\left\{\int_{\beta}^{0} \frac{d \kappa}{\sqrt{(\alpha-\kappa)(\kappa-\beta)}}+\int_{0}^{\alpha} \frac{d \kappa}{\sqrt{(\alpha-\kappa)(\kappa-\beta)}}\right\} \\
& \leq \frac{4 \sqrt{d}}{n \alpha^{2}}\left\{\frac{1}{\sqrt{\alpha}}[2 \sqrt{(\kappa-\beta)}]_{\beta}^{0}+\frac{1}{\sqrt{-\beta}}[-2 \sqrt{(\alpha-\kappa)}]_{0}^{\alpha}\right\} \\
& =\frac{8 \sqrt{d}}{n \alpha^{2}}\left\{\frac{\sqrt{-\beta}}{\sqrt{\alpha}}+\frac{\sqrt{\alpha}}{\sqrt{-\beta}}\right\}=O\left(\frac{1}{\alpha}\right)=O\left(\frac{1}{\sqrt[4]{d}}\right) .
\end{aligned}
$$

Thus, since the angular progression $\Lambda^{\lambda}(d)$ varies continuously as $d$ moves in $\left(d_{o}, \infty\right)$, its range of variation contains the interval

$$
\left(-\frac{4 \pi \sqrt{d_{o}} \kappa_{o}}{\left(\lambda+2 \kappa_{o}\right) \sqrt{6 \kappa_{o}^{2}+4}}, 0\right)
$$

(for a numerical estimation, see Figure 4). This finishes the proof.

Proposition 3.2 can be used in combination with (2.8) and (3.1) to obtain explicit parameterizations of closed critical points of $\mathcal{F}^{\lambda}$ in the unit 2-sphere. See Figures 1-3 and Section 4.

As for the stability of the critical curves, the second variation formula for (1.1) can also be computed following [2]. However, its application to the stability analysis of the critical curves is quite limited due to its complexity. Nevertheless, there is one case in which this analysis can be fulfilled: critical circles. We say that a critical curve $\gamma$ of $\mathcal{F}^{\lambda}$ is stable if, for any variation $\gamma(w, s)$ of the curve, $\left.\left(d^{2} \mathcal{F}^{\lambda}(\gamma(w, s)) / d w^{2}\right)\right|_{w=0} \geq 0$ is satisfied.

As already observed, circles $\gamma_{\epsilon}$ of curvature $\epsilon$, where $\epsilon=\kappa_{o}$ is given in (2.3), are critical points of $\mathcal{F}^{\lambda}$. Denote by $\gamma_{\epsilon}^{m}$ the $m$-covering of $\gamma_{\epsilon}$. Then, according to Proposition 6 of [2], $\gamma_{\epsilon}^{m}$ will be stable if and only if the two conditions

$$
P^{\prime \prime}(\epsilon)>0, \quad \Theta^{\lambda}(\epsilon, m) \leq 1,
$$



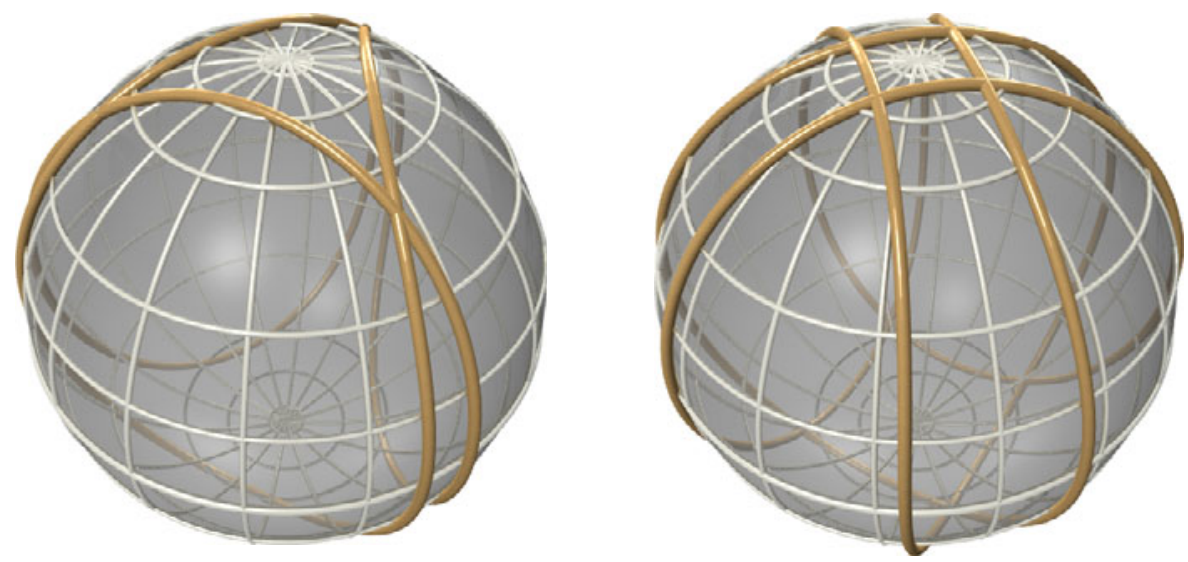

FIGURE 1. Closed extremals for $\mathcal{F}^{1 / 4}$ : these close up in 3 and 4 curvature periods, respectively.
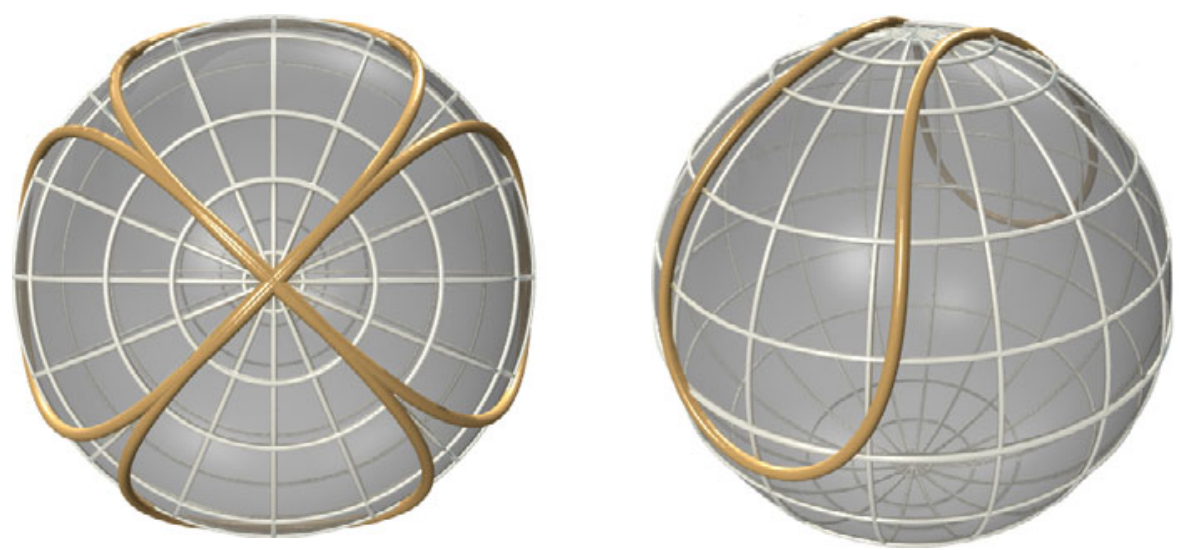

FIGURE 2. Closed extremals for $\mathcal{F}^{4}$ : these close up in 4 and 2 curvature periods, respectively.

are satisfied, where

$$
\Theta^{\lambda}(\epsilon, m):=\left|m\left(1-\sqrt{1-\frac{P(\epsilon)}{P^{\prime \prime}(\epsilon)\left(\epsilon^{2}+1\right)^{2}}}\right)\right|,
$$

and $P(x)=x^{2}+\lambda x$. In our case $P^{\prime \prime}(\epsilon)=2$ for any $\lambda$ and lengthly but straightforward computations show that $\Theta^{\lambda}(\epsilon, 1)$ will always be less than 1 . To summarize, we state the following proposition.

PROPOSITION 3.3. For any $\lambda>0$, the once covered circle $\gamma_{\epsilon}$ with curvature (2.3) is stable. 

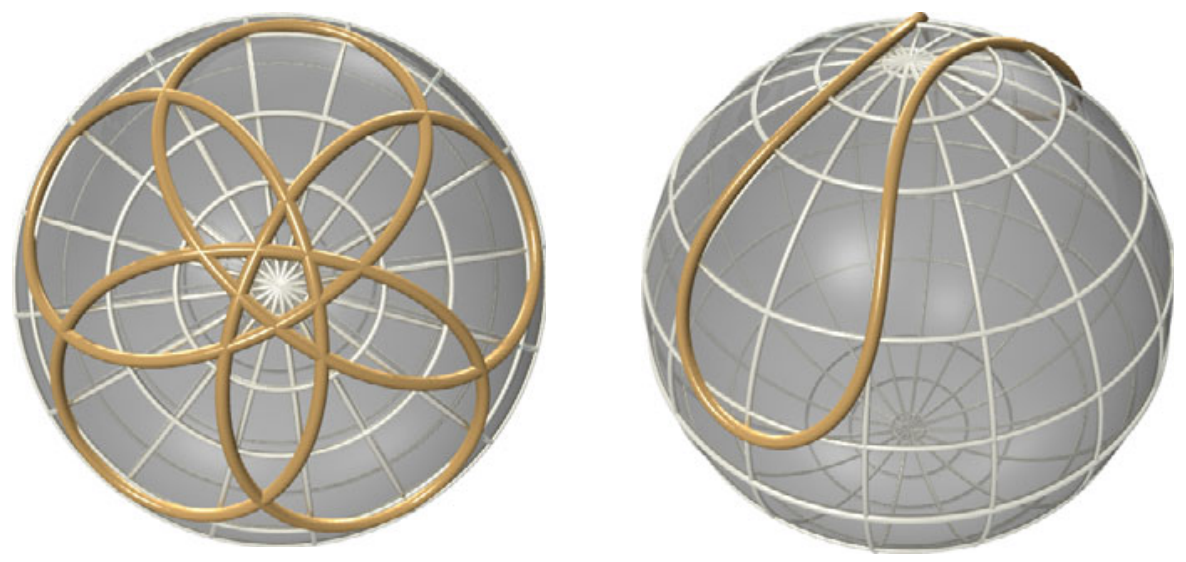

FIGURE 3. Closed extremals for $\mathcal{F}^{10}$ : these close up in 5 and 2 curvature periods, respectively.



FIGURE 4. Variations of $\Lambda^{\lambda}(d)$ for various values of $\lambda$.

\section{Numerical experiments}

In connection with the angular progression $\Lambda^{\lambda}(d)$ and the statement of Proposition 3.2, a couple of interesting questions remain open. The first concerns the potential existence of other closed critical curves of $\mathcal{F}^{\lambda}$, not included in Proposition 3.2. The second is related to the uniqueness of the closed critical curve (up to multiple coverings of the $\left.\gamma_{m, n}(s)\right)$ associated with any pair of natural numbers $n, m$ satisfying the conditions of Proposition 3.2. Our numerical experiments suggest that both questions can be answered in the positive. Figure 4 shows the angular progression for different values of $\lambda$. The angular progression for $\lambda=0.01$ and $\lambda=0.1$ can be seen at the bottom of the picture, while the curve at the top corresponds to $\lambda=100$. In between are curves for $\lambda=1,2,3,4,5,6,7,8,9,10,20,30,40,50$. This figures illustrates 


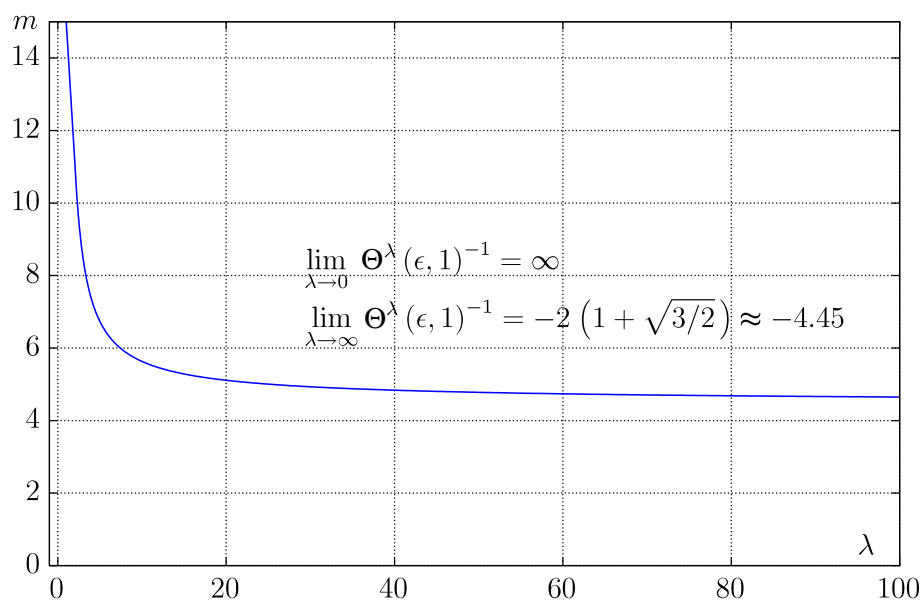

FIGURE 5. Stable $m$-coverings of $\gamma_{\epsilon}$.



FIGURE 6. Number of periods of closed extremals.

the answer to the first question, since it indicates that $\Lambda^{\lambda}(d)$ varies continuously in $\left(-4 \pi \sqrt{d_{o}} \kappa_{o} /\left(\left(\lambda+2 \kappa_{o}\right) \sqrt{6 \kappa_{o}^{2}+4}\right), 0\right)$ as $d$ moves in $\left(d_{o}, \infty\right)$.

As for the second question, monotonicity of $\Lambda^{\lambda}(d)$ in terms of $d$, for a fixed $\lambda>0$, would imply a positive answer to it. It seems very involved and difficult to prove this fact. Even for $\lambda=0$ it is a formidable task [13]. Nevertheless, our numerical computations (see Figure 4) suggest that this is apparently the case for any $\lambda>0$.

On the other hand, in order to obtain explicit parameterizations of the closed critical curves for $\mathcal{F}^{\lambda}$, we need first to find a real number $d>0$ and natural numbers 


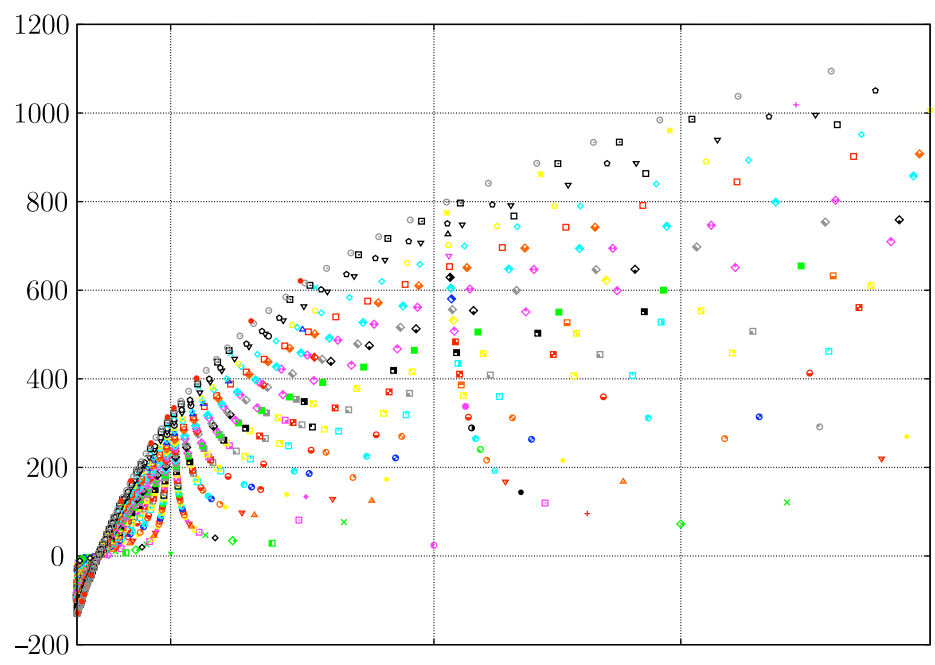

FIGURE 7. Energy values of closed extremals.

$m, n$ satisfying the conditions of Propositions 3.2 and 3.1. Then we have to use (2.7)-(2.8) to obtain their curvature, substitute in (3.1) and, finally, integrate the corresponding equations. Of course, this is not possible in general, but the procedure can be implemented by a numerical algorithm allowing us to graphically obtain these examples. Figures 1-3 show the outcome of the algorithm in a few cases.

Another interesting question has to do with the stability. The value for the number of stable coverings given in Proposition 3.3 is quite conservative $(m=1)$. Figure 5 shows an estimation, depending on $\lambda$, of the maximum number of coverings of the critical circle $\gamma_{\epsilon}$ which are stable. It indicates that the number of stable coverings increases as $\lambda$ approaches 0 and that 4-coverings are stable for any $\lambda$.

Our final experiments are shown in Figures 6 and 7. Figure 6 shows the number of closed critical curves which close up in less than 600 periods of their curvatures. For a given $\lambda$, the dots on the vertical line $x=\lambda$ correspond to closed critical curves for $\mathcal{F}^{\lambda}(\gamma)$. For any dot, its height on the vertical axis gives the number of periods needed for the critical curve associated with it to close up. Apparently, although for any $\lambda>0$ there exists a two-parameter family of closed critical curves, there are certain values of $\lambda$ for which the extremal curves with nonconstant curvature require a huge number of periods of their curvature to close up. Figure 7 shows the energy value $\mathcal{F}^{\lambda}(\gamma)$ for the closed extremals considered in Figure 6, when evaluated over the arc of curve corresponding to just one period of their curvature.

\section{References}

[1] J. Arroyo, M. Barros and O. J. Garay, 'Some examples of critical points for the total mean curvature functional', Proc. Edinb. Math. Soc. 43 (2000), 587-603. 
[2] J. Arroyo, O. J. Garay and J. J. Mencía, 'Closed generalized elastic curves in $\mathbb{S}^{2}(1)$ ', J. Geom. Phys. 48 (2003), 339-353.

[3] J. Arroyo, O. J. Garay and J. J. Mencía, 'Extremals of curvature energy actions on spherical closed curves', J. Geom. Phys. 51 (2004), 101-125.

[4] J. Arroyo, O. J. Garay and J. J. Mencía, 'Elastic circles in 2-spheres', J. Phys. A.: Math. Gen. 39 (2006), 2307-2324.

[5] M. Barros, 'Simple geometric models with applications to physics', in: Curvature and Variational Modeling in Physics and Biophysics, AIP Conference Proceedings, 1002, (eds. O. J. Garay, E. García-Río and R. Vázquez-Lorenzo) (AIP, Melville, NY, 2008), pp. 71-113.

[6] R. Bryant and P. Griffiths, 'Reduction of order for constrained variational problems and $\int_{\gamma} \frac{\kappa^{2}}{2} d s^{\prime}$, Amer. J. Math. 108 (1986), 525-570.

[7] I. S. Gradsteyn and I. M. Ryzhik, Table of Integrals, Series, and Products (Academic Press, New York, 1980).

[8] V. Jurdjevic, 'Non-Euclidean elastica', Amer. J. Math. 117 (1995), 93-124.

[9] A. A. Kapustnikov, A. Pashnev and A. Pichugin, 'Canonical quantization of the kink model beyond the static solution', Phys. Rev. D 55 (1977), 2257-2264.

[10] S. Kawakubo, 'Kirchhoff elastic rods in the three-dimensional space forms', J. Math. Soc. Japan 60 (2008), 551-582.

[11] N. Koiso, 'Elasticae in a Riemannian manifold', Osaka J. Math. 29 (1992), 539-543.

[12] L. Landau and E. Lifshitz, Theory of Elasticity, Course of Theoretical Physics, 7 (ButterworthHeinemann, Boston, MA, 1986).

[13] J. Langer and D. A. Singer, 'The total squared curvature of closed curves', J. Differential Geom. 20 (1984), 1-22.

[14] J. Langer and D. A. Singer, 'Knotted elastic curves in $\mathbb{R}^{3}$, J. Lond. Math. Soc. 16 (1984), $512-520$.

[15] J. Langer and D. A. Singer, 'Curve-straightening in Riemannian manifolds', Ann. Global Anal. Geom. 5(2) (1987), 133-150.

[16] A. Linner, 'Curve straightening and the Palais-Smale condition', Trans. Amer. Math. Soc. 350 (1998), 3743-3765.

[17] L. Noakes and T. Popiel, 'Elastica in SO(3)', J. Aust. Math. Soc. 83(1) (2007), 105-124.

[18] M. S. Plyushchay, 'Massless particle with rigidity as a model for description of bosons and fermions', Phys. Lett. B 243 (1990), 383-388.

JOSU ARROYO, Department of Mathematics, Faculty of Science and Technology, University of the Basque Country, Aptdo 644, 48080 Bilbao, Spain e-mail: josujon.arroyo@ehu.es

ÓSCAR J. GARAY, Department of Mathematics, Faculty of Science and Technology, University of the Basque Country, Aptdo 644, 48080 Bilbao, Spain e-mail: oscarj.garay@ehu.es

JOSE MENCÍA, Department of Mathematics, Faculty of Science and Technology, University of the Basque Country, Aptdo 644, 48080 Bilbao, Spain e-mail: jj.mencia@ehu.es 